\title{
Association between pro- and anti- inflammatory cytokines and depressive symptoms in patients with diabetes- potential differences by diabetes type and depression scores
}

Christian Herder $\mathbb{1}^{1,2}$, Andreas Schmitt 2,3 , Florian Budden ${ }^{1,2}$, André Reimer ${ }^{2,3}$, Bernhard Kulzer, ${ }^{2,3,4}$, Michael Roden ${ }^{1,2,5}$, Thomas Haak ${ }^{2,3,4}$ and Norbert Hermanns $s^{2,3,4}$

\begin{abstract}
Subclinical inflammation has been implicated in the development of depression, a common comorbidity of type 1 diabetes (T1D) and type 2 diabetes (T2D). This study aimed to characterise the relationships between biomarkers of inflammation and depressive symptoms in T1D and T2D. Biomarkers of inflammation were measured in serum of participants with elevated depressive symptoms and T1D ( $n=389$, mean age 38 years, diabetes duration $15 \pm 11$ years) or T2D ( $n=204$, mean age 56 years, diabetes duration $13 \pm 8$ years). Subclinical depression was examined using three questionnaires (Center for Epidemiologic Studies Depression [CES-D], Patient Health Questionnaire-9 [PHQ-9], 5-item World Health Organization Well-Being Index [WHO-5]). In T1D, levels of interleukin-1 receptor antagonist (IL-1RA) were positively associated with two depression scores (CES-D, PHQ-9), and high-sensitivity C-reactive protein (hsCRP) was positively associated with depression for one score (WHO-5) after adjustment for age, sex, body mass index, diabetes duration, metabolic variables, medication and comorbidities $(P=0.008-0.042)$. In T2D, IL-18 and IL-1RA were positively associated with depression for two scores (IL-18: PHQ-9, WHO-5; IL-1RA: CES-D, WHO-5), hsCRP was associated with one depression score (PHQ-9), and adiponectin showed an inverse association with one depression score (PHQ-9) after adjustment $(P=0.006-0.048)$. No associations were found for IL-6 and CC-chemokine ligand 2 (CCL2). In conclusion, we observed associations between hsCRP, IL-1RA and depressive symptoms in patients with diabetes. In T2D, there was additional evidence for associations of IL-18 and (inversely) adiponectin with depressive symptoms. The strength of the associations appeared to depend on diabetes type and the method used to asssess depressive symptoms.
\end{abstract}

\section{Introduction}

Depression is one of the most frequent comorbidities in both patients with type 1 diabetes (T1D) and type 2

\footnotetext{
Correspondence: Christian Herder (christian.herder@ddz.uni-duesseldorf.de) ${ }^{1}$ Institute for Clinical Diabetology, German Diabetes Center, Leibniz Center for Diabetes Research at Heinrich Heine University Düsseldorf, Düsseldorf, Germany

${ }^{2}$ German Center for Diabetes Research (DZD), München-Neuherberg, Germany Full list of author information is available at the end of the article
}

diabetes $(\mathrm{T} 2 \mathrm{D})^{1-4}$. The high prevalence of depressive disorders in patients with diabetes represents an important clinical problem ${ }^{5}$ because not only major depression but also lower levels of depressive symptoms are linked with poorer long-term prognosis (e.g., increased risk of diabetes-related micro- and macrovascular complications) and higher mortality ${ }^{6-10}$.

Subclinical inflammation has been implicated as important pathomechanism in patients with the double 
burden of diabetes and depression ${ }^{11-14}$. Inflammatory processes contribute to the development of diabetes, although it is important to note that different types of immune activation are involved in the aetiology and pathomechanisms leading to $\mathrm{T} 1 \mathrm{D}$ and $\mathrm{T} 2 \mathrm{D}^{15,16}$. In addition, several lines of evidence have also linked inflammation and depression ${ }^{12,14,17-19}$. These include mechanistic studies in animals and clinical studies, but also cross-sectional and prospective epidemiological studies showing that high systemic levels of cytokines and chemokines are associated with a higher prevalence or risk of subclinical and major depression ${ }^{20-22}$.

Most of these latter studies are based on samples from the general population, whereas only few studies addressed the association between biomarkers of inflammation and depression specifically in patients with $\mathrm{T}_{1} \mathrm{D}^{23,24}$ or T2D ${ }^{23-27}$. Therefore, it is currently not well understood (i) if subclinical inflammation is associated with depressive symptoms in patients with diabetes, (ii) if associations differ by diabetes type and (iii) to what extent associations are independent from confounders such as glycaemic control, diabetes duration, medication use or diabetesrelated comorbidities. In addition, the aforementioned studies used different methods to diagnose depressive symptoms, which might have influenced study results.

Thus, the major aim of this study was to test the hypothesis that biomarkers of subclinical inflammation are associated with depressive symptoms in a large sample of individuals with T1D and T2D. We used three different questionnaire-based scores to assess depressive symptoms, so that we were able to compare such associations not only between diabetes types, but also between depression measurements.

\section{Study population and methods Study population}

This study used baseline data from two single centre randomised clinical trials with comparable study populations which were conducted at the Diabetes Center Mergentheim (a specialised inpatient diabetes care centre) and tested interventions for depressive symptoms in patients with diabetes (ClinicalTrials.gov Identifiers NCT01009138 and NCT01812291). Both studies were approved by the Ethics Committee of the State Medical Chamber of Baden-Württemberg, Germany (DIAMOS: 2009-034-f, ECCE-HOMO: F-2013-011), and performed according to the Declaration of Helsinki. All participants provided written informed consent.

The first study tested a self-management-oriented group programme (DIAMOS (Strengthening Diabetes Motivation)) which used cognitive behavioural interventions aiming at the reduction of diabetes distress. As described, patients were recruited at an inpatient diabetes centre with the following inclusion criteria: diabetes mellitus, elevated depressive symptoms (Center for Epidemiologic Studies Depression (CES-D] score) $\geq 16$ ), age 18-70 years and sufficient German language skills. Exclusion criteria were major depression, schizophrenia/ psychotic disorder, severe eating disorder, bipolar disorder, addictive disorder or personality disorder, current use of antidepressant medication or ongoing psychotherapy, being bedridden, or being under guardianship $^{28}$.

The second study aimed to test the efficacy of a stepcare approach to manage depression in diabetic patients and putative inflammatory mechanisms between diabetes and depression (acronym: ECCE-HOMO). Inclusion criteria were diabetes mellitus, elevated depressive symptoms (CES-D > 16) and/or elevated diabetes-related distress (Problem Areas in Diabetes Questionnaire (PAID) $>40$ ), age 18-70 years, and sufficient German language skills. Exclusion criteria comprised severe clinical depression according to ICD-10 (F32.2/F32.3), suicidal ideation, severe somatic illness (e.g., end-stage diabetes complication, terminal stage cancer) or dementia. Further exclusion criteria were current psychotherapeutic/psychiatric treatment, current antidepressive medication, schizophrenia/psychotic disorder, severe eating disorder, bipolar disorder, addictive disorder, personality disorder, being bedridden, or being under guardianship.

\section{Assessment of depressive symptoms}

Patients underwent a psychiatric interview (Composite International Diagnostic Interview/CIDI $)^{29}$ to exclude patients with severe comorbid mental diseases, bipolar depressive disorders and major depression with suicidal ideation. Depressive symptoms were assessed by using three validated questionnaires which were filled in by the study participants. The questionnaires were given to the patients in the order listed below by an experienced clinical psychologist with precise instructions to the patients and with the opportunity to clarify any questions. After informed consent patients completed the questionnaires.

The baseline examinations of the DIAMOS and ECCEHOMO studies included the following questionnaires to assess depressive symptoms and well-being:

- The German version of the CES-D ${ }^{30,31}$, a sensitive instrument to detect depressive symptoms within the previous week and to monitor changes over time; ${ }^{32,33}$

- The Patient Health Questionnaire-9 (PHQ-9), a depression scale to assess the frequency of each of the nine symptoms of major depression as defined by the DSM-V, which is also able to make a criteriabased diagnosis of major depression ${ }^{34}$, and

- The 5-item World Health Organization Well-Being Index (WHO-5) to assess subjective psychological well-being with adequate validity both as a screening 
tool for depression and as an outcome measure in clinical trials ${ }^{35}$.

CES-D and PHQ-9 scores range from 0-60 to 0-27 points, respectively, with higher scores indicating higher depressive symptoms. The WHO-5 score ranges from 0 to 25 with lower scores indicating lower well-being and more depressive symptoms. For all three questionnaires, we used the continuous scores to make optimal use of the variation in symptoms.

\section{Measurement of biomarkers of subclinical inflammation}

Fasting blood samples were taken between 0630 and 0800 hours according to standard operating procedures on the next working day following the day when depressive symptoms were assessed. Biomarkers of subclinical inflammation were measured in serum samples at the German Diabetes Center as described ${ }^{28,36}$. The same assays and control sera were used for samples from both study populations. Fasting serum levels of hsCRP were measured on a Roche/Hitachi c 311 analyzer (Basel, Switzerland). Fasting serum levels of IL-6, IL-1RA, CCchemokine ligand 2 (CCL2, also known as monocyte chemotactic protein-1/MCP-1) and total adiponectin were determined using Quantikine HS (IL-6) or Quantikine (IL-1RA, MCP-1, adiponectin) ELISA kits from R\&D systems (Wiesbaden, Germany). Serum IL-18 was measured using the ELISA kit from MBL (Nagoya, Japan). These six biomarkers were chosen because of their associations with depressive symptoms and/or depression in the general population ${ }^{20-27,37-39}$ and their additional relationship with other diabetic comorbidities and complications $^{40-45}$.

\section{Assessment of confounding variables}

Demographic, anthropometric and clinical data were available from detailed baseline examinations of both trials ${ }^{28}$.

Information about medication was obtained from medical records. Lipid-lowering drugs included atorvastatin, fluvastatin, pitavastatin, lovastatin, pravastatin, rosuvastatin, simvastatin and ezetimibe. Non-steroidal anti-inflammatory drugs (NSAIDs) included diclofenac, ibuprofen and naproxen. Antithrombotic medication included clopidogrel, phenprocoumon, enoxaparin and aspirin. Antidepressant medication included selective serotonin reuptake inhibitors, serotonin-norepinephrine reuptake inhibitors, tricyclic antidepressants, monoamine oxidase inhibitors, reversible monoamine oxidase A inhibitors, tetracyclic antidepressants, noradrenergic and specific serotonergic antidepressant and St John's wort.

The diagnosis of diabetic nephropathy was based on a measured glomerular filtration rate of $<60 \mathrm{ml} / \mathrm{min} /$ $1.73 \mathrm{~m}^{2}$. Diabetic retinopathy was diagnosed by ophthalmologic examination or based on previous laser coagulation treatment. Diagnoses of cardiovascular disease, myocardial infarction, stroke or peripheral arterial occusive disease (PAOD) were made based on previous events or revascularisation measures.

\section{Statistical analysis}

Analyses were based on 389 individuals with T1D and 204 individuals with T2D with a complete core data set comprising age, sex, body mass index (BMI), diabetes type, diabetes duration, comorbidities and medication (DIAMOS, $n=223$ T1D and $n=114$ T2D; ECCEHOMO, $n=166$ T1D and $n=90$ T2D). Data from both studies were pooled, and analyses were then stratified by diabetes type because of well-known differences in the pathophysiology including levels of biomarkers of subclinical inflammation between T1D and T2D.

Data are presented as mean (SD), median (25th and 75th percentiles) or percentages (\%) for continuous or categorical variables. Differences between diabetes types were tested using Student's $t$-test (two-sided) or Pearson's $\mathrm{Chi}^{2}$ test. Unadjusted correlations between biomarkers of subclinical inflammation (ln-transformed to approximate normal distribution) and/or depression scores were estimated using Pearson's correlation coefficients $r$ and corresponding $P$ values. Multivariate associations between biomarkers of subclinical inflammation (independent variables, separate models for $\ln$-transformed serum levels of each biomarker) and depression scores as outcome (dependent variables, separate models for each score) were estimated using multivariable linear regression models with increasing complexity: model 1: adjusted for age, sex and study; model 2, model $1+$ adjustment for BMI, haemoglobin A1c (HbA1c), time since diagnosis of diabetes, total cholesterol, triglycerides, use of lipidlowering drugs, hypertension, use of NSAIDs, use of antithrombotic medication, use of antidepressant medication; model 3: model 2 +adjustment for number of microvascular comorbidities and number of macrovascular comorbidities. Adjustment for the time of blood sampling was not necessary given the narrow timewindow in which blood samples were taken. Regression coefficients $\beta$ are standardised to 1SD of $\ln$-transformed serum levels of biomarkers of subclinical inflammation.

In the meta-analysis from Howren et al. ${ }^{20}$ effect sizes of the associations between biomarkers of inflammation and depression ranged between $d=0.15$ and $d=0.35$. The sample size of 204 participants with T2D and 389 participants with T1D allowed the detection of significant associations between biomarkers of inflammation and depressive symptoms of a small to moderate effect size of $d=0.20$ with a power of $82 \%$ in people with T2D and of $97 \%$ in people with T1D.

We considered $P$ values of $<0.05$ to indicate statistically significant differences or associations. All analyses were performed using Systat 12.0 and SPSS 23. 
Table 1 Study population stratified by diabetes type

\begin{tabular}{|c|c|c|c|c|c|}
\hline Variable & $T 1 D$ & $n$ & $T 2 D$ & $n$ & $P$ \\
\hline Age (years) & $38.1(13.1)$ & 389 & $55.8(9.1)$ & 204 & $<0.001$ \\
\hline Sex (men/women) (\%) & $41.6 / 58.4$ & 389 & $56.9 / 43.1$ & 204 & $<0.001$ \\
\hline $\mathrm{BMI}\left(\mathrm{kg} / \mathrm{m}^{2}\right)$ & $26.0(4.6)$ & 389 & $35.3(6.6)$ & 204 & $<0.001$ \\
\hline $\mathrm{HbA1c}(\%)$ & $8.2(7.4 ; 9.3)$ & 387 & $8.9(8.0 ; 10.3)$ & 202 & $<0.001$ \\
\hline $\mathrm{HbA1c}(\mathrm{mmol} / \mathrm{mol})$ & $66(57 ; 78)$ & 387 & $74(64 ; 89)$ & 202 & $<0.001$ \\
\hline Time since diagnosis of diabetes (years) & $15.2(11.2)$ & 389 & $12.6(7.6)$ & 204 & 0.001 \\
\hline Total cholesterol (mg/dl) & $190(173 ; 226)$ & 386 & $186(161 ; 225)$ & 202 & 0.895 \\
\hline Triglycerides (mg/dl) & $93(71 ; 138)$ & 386 & $180(130 ; 273)$ & 202 & $<0.001$ \\
\hline Lipid-lowering drugs (\%) & 11.8 & 389 & 45.6 & 204 & $<0.001$ \\
\hline Hypertension (\%) & 23.9 & 389 & 75.0 & 204 & $<0.001$ \\
\hline NSAIDs (\%) & 2.1 & 389 & 2.9 & 204 & 0.524 \\
\hline Antithrombotic drugs (\%) & 5.9 & 389 & 42.6 & 204 & $<0.001$ \\
\hline Number of diabetes-related comorbidities & $0.5(0.8)$ & 389 & $1.5(1.4)$ & 204 & $<0.001$ \\
\hline Retinopathy (\%) & 20.6 & 389 & 25.5 & 204 & 0.182 \\
\hline Nephropathy (\%) & 4.1 & 389 & 18.6 & 204 & $<0.001$ \\
\hline Polyneuropathy (\%) & 15.2 & 389 & 56.4 & 204 & $<0.001$ \\
\hline Diabetic foot (\%) & 1.0 & 389 & 6.9 & 204 & 0.002 \\
\hline PAOD (\%) & 1.6 & 389 & 11.0 & 204 & $<0.001$ \\
\hline Coronary heart disease (\%) & 1.5 & 389 & 18.1 & 204 & $<0.001$ \\
\hline Myocardial infarction (\%) & 0.5 & 389 & 6.9 & 204 & 0.001 \\
\hline Stroke (\%) & 1.0 & 389 & 4.9 & 204 & 0.016 \\
\hline CES-D & $21.0(10.8)$ & 379 & $21.5(10.6)$ & 196 & 0.568 \\
\hline PHQ-9 & $8.9(5.0)$ & 380 & $9.3(5.2)$ & 197 & 0.317 \\
\hline WHO-5 & $10.2(5.7)$ & 379 & $11.0(6.0)$ & 197 & 0.170 \\
\hline Antidepressant drugs (\%) & 1.3 & 389 & 4.4 & 204 & 0.045 \\
\hline $\mathrm{hsCRP}(\mathrm{mg} / \mathrm{dl})$ & $0.11(0.06 ; 0.28)$ & 376 & $0.30(0.13 ; 0.73)$ & 195 & $<0.001$ \\
\hline IL-6 (pg/ml) & $1.0(0.7 ; 1.7)$ & 376 & $2.4(1.5 ; 3.6)$ & 195 & $<0.001$ \\
\hline IL-18 (pg/ml) & $219(178 ; 280)$ & 376 & $274(203 ; 354)$ & 195 & $<0.001$ \\
\hline CCL2 (pg/ml) & $488(376 ; 650)$ & 376 & $529(423 ; 766)$ & 195 & 0.001 \\
\hline IL-1RA (pg/ml) & $351(264 ; 504)$ & 376 & $727(478 ; 1160)$ & 195 & $<0.001$ \\
\hline Adiponectin (ng/ml) & $9879(6251 ; 15,392)$ & 376 & $3927(2635 ; 5954)$ & 195 & $<0.001$ \\
\hline
\end{tabular}

$B M I$ body mass index, CCL2 CC-chemokine ligand 2, CES-D Center for Epidemiologic Studies Depression Scale, hsCRP high-sensitivity C-reactive protein, IL interleukin, IL-1RA IL-1 receptor antagonist, NSAIDs non-steroidal anti-inflammatory drugs, PAOD peripheral arterial occlusive disease, PHQ-9 Patient Health Questionnaire, T1D type 1 diabetes, T2D type 2 diabetes, WHO-5 5-item World Health Organization Well-Being Index

Continuous data are given as mean (SD) or median (25th percentile; 75th percentile), categorial variables are given as percentages (\%)

Diabetes-related comorbidities include retinopathy, nephropathy, polyneuropathy, diabetic foot, PAOD, coronary heart disease, myocardial infarction and stroke (max. 8)

\section{Results}

\section{Study population}

The characteristics of the study population stratified by diabetes type are summarised in Table 1. Patients with T1D were younger, but had a longer time since the diagnosis of diabetes than patients with T2D. In addition, they were more often female, had lower levels of BMI, HbA1c and triglycerides than patients with T2D, whereas no group difference was found for total cholesterol. Patients with T1D were also less frequently hypertensive, less likely to use lipid-lowering and antithrombotic drugs and suffered less often from diabetes-related 
Table 2 Univariate correlations between biomarkers of subclinical inflammation and depression scores in individuals with T1D (upper right) and T2D (lower left)

\begin{tabular}{llllllllll}
\hline Variable & CES-D & PHQ-9 & WHO-5 & hsCRP & IL-6 & IL-18 & CCL2 & IL-1RA & Adiponectin \\
\hline CES-D & - & $0.806^{* * *}$ & $-0.750^{* * *}$ & 0.043 & 0.051 & -0.035 & $0.138^{* *}$ & $0.160^{* *}$ & 0.051 \\
PHQ-9 & $0.811^{* * *}$ & - & $-0.703^{* * *}$ & 0.069 & 0.072 & 0.009 & 0.056 & $0.144^{* *}$ & 0.101 \\
WHO-5 & $-0.712^{* * *}$ & $-0.701^{* * *}$ & - & $-0.141^{* *}$ & -0.071 & 0.043 & -0.087 & $-0.138^{* *}$ & -0.082 \\
hSCRP & $0.192^{* *}$ & $0.176^{*}$ & $-0.176^{*}$ & - & $0.377^{* * *}$ & 0.094 & -0.049 & $0.384^{* * *}$ & $-0.135^{* *}$ \\
IL-6 & 0.036 & 0.009 & -0.011 & $0.606^{* * *}$ & - & 0.076 & $0.207^{* * *}$ & $0.375^{* * *}$ & $-0.163^{* *}$ \\
IL-18 & 0.046 & 0.064 & -0.130 & $0.403^{* * *}$ & $0.334^{* * *}$ & - & $-0.115^{*}$ & 0.074 & -0.076 \\
CCL2 & 0.130 & 0.098 & -0.085 & 0.049 & $0.198^{* *}$ & 0.015 & - & $0.104^{*}$ & 0.033 \\
IL-1RA & $0.212^{* *}$ & $0.150^{*}$ & $-0.233^{* *}$ & $0.440^{* * *}$ & $0.450^{* * *}$ & $0.348^{* * *}$ & $0.249^{* * *}$ & - & $-0.303^{* * *}$ \\
Adiponectin & -0.102 & -0.134 & 0.065 & -0.064 & -0.093 & -0.088 & -0.019 & -0.115 & - \\
\hline
\end{tabular}

Data are given as Pearson correlation coefficients $r .{ }^{*} P<0.05 ;{ }^{* *} P<0.01 ;{ }^{* * *} P<0.001$. Circulating levels of biomarkers of subclinical inflammation were In-transformed

comorbidities compared to patients with T2D. No differences were observed between both groups regarding their use of NSAIDs and their CES-D, PHQ-9 and WHO5 scores, but patients with T2D showed a slightly higher prevalence of antidepressant drug use. Serum levels of hsCRP, IL-6, IL-18, CCL2 and IL-1RA were lower, whereas adiponectin was higher in T1D than in T2D.

As shown in Table 2, the pairwise correlations between the CES-D, PHQ-9 and WHO-5 scores were similar among patients with T1D and T2D with correlation coefficients $|r|$ between 0.70 and 0.81 (all $P<0.001$ ). Positive pairwise correlations of the biomarkers of inflammation were mainly observed for hsCRP, IL-6, IL-18 and IL-1RA with stronger associations in T2D (r between 0.33 and 0.61 , all $P<0.001$ ) than in T1D ( $\mathrm{r}$ between 0.07 and $0.38, P$ between $<0.001$ and 0.155 ) (Table 2). Correlations between CCL2 and the aforementioned biomarkers were less pronounced in both diabetes types. Adiponectin levels showed inverse associations with hsCRP, IL-6 and IL-1RA in T1D (all $P<$ 0.01 ), whereas no significant correlations were found in T2D.

\section{Associations between biomarkers of inflammation and depressive symptoms in patients with T1D}

Unadjusted analyses revealed positive correlations between biomarkers of inflammation and depressive symptom scores for hsCRP (WHO-5), CCL2 (CES-D) and IL-1RA (CES-D, PHQ-9, WHO-5) in patients with T1D (Table 2). Please note that a high level of depressive symptoms is indicated by high CES-D and PHQ-9 scores, but low WHO-5 scores, so that inverse correlations between biomarkers and WHO- 5 correspond to positive correlations between biomarkers and CES-D or PHQ-9.

The associations between hsCRP and the WHO- 5 score and between IL-1RA and all three depression scores persisted after adjustment for age, sex and study cohort (Table 3, model 1). After further adjustment for BMI, metabolic variables, medication use and diabetes-related comorbidities, hsCRP remained associated with the WHO-5 score, and IL-1RA remained associated with the CES-D and PHQ-9 scores (Table 3, models 2 and 3).

No significant associations were observed between IL-6, IL-18 and adiponectin and any of the three scores at all levels of adjustment (Tables 2 and 3).

\section{Associations between biomarkers of inflammation and depressive symptoms in patients with T2D}

In patients with T2D, hsCRP and IL-1RA levels were positively correlated with depressive symptoms assessed by all three scores in the unadjusted analyses, whereas no significant correlations were found for IL-6, IL-18, CCL2 and adiponectin (Table 2).

Adjustment for age, sex and the other covariables in models 1-3 strengthened some of the aforementioned associations, whereas others were attenuated (Table 4). In the fully adjusted model 3 , high levels of hsCRP were associated with depressive symptoms in one score (PHQ9), IL-18 with two scores (PHQ-9, WHO-5), IL-1RA also with two scores (CES-D, WHO-5) and adiponectin with one score (PHQ-9). IL-6 and CCL2 levels were not related to depressive symptoms in any model.

\section{Discussion}

Our study revealed several novel associations between biomarkers of subclinical inflammation and depressive symptoms in patients with diabetes. In T1D, higher levels of hsCRP and IL-1RA were associated with more pronounced depressive symptoms. In T2D, higher levels of hsCRP, IL-18 and IL-1RA and lower adiponectin levels were associated with higher depressive symptoms. These associations were independent of multiple confounders, 
Table 3 Associations between biomarkers of subclinical inflammation and depression scores in individuals with T1D

\begin{tabular}{|c|c|c|c|c|c|c|c|}
\hline \multirow[t]{2}{*}{ Biomarker } & \multirow[t]{2}{*}{ Model } & \multicolumn{2}{|l|}{ CES-D } & \multicolumn{2}{|l|}{ PHQ-9 } & \multicolumn{2}{|l|}{ WHO-5 } \\
\hline & & $\beta$ & $P$ & $\beta$ & $P$ & $\beta$ & $P$ \\
\hline \multirow[t]{3}{*}{ hsCRP } & 1 & 0.015 & 0.769 & 0.038 & 0.470 & -0.113 & 0.030 \\
\hline & 2 & 0.029 & 0.627 & 0.046 & 0.456 & -0.129 & 0.034 \\
\hline & 3 & 0.025 & 0.684 & 0.044 & 0.471 & -0.123 & 0.042 \\
\hline \multirow[t]{3}{*}{ IL-6 } & 1 & 0.035 & 0.504 & 0.072 & 0.174 & -0.064 & 0.220 \\
\hline & 2 & 0.026 & 0.636 & 0.066 & 0.235 & -0.054 & 0.325 \\
\hline & 3 & 0.022 & 0.686 & 0.068 & 0.225 & -0.049 & 0.374 \\
\hline \multirow[t]{3}{*}{ IL-18 } & 1 & 0.027 & 0.601 & 0.062 & 0.230 & -0.017 & 0.741 \\
\hline & 2 & -0.002 & 0.966 & 0.035 & 0.512 & 0.016 & 0.763 \\
\hline & 3 & -0.007 & 0.893 & 0.029 & 0.588 & 0.020 & 0.701 \\
\hline \multirow[t]{3}{*}{ CCL2 } & 1 & 0.030 & 0.570 & 0.006 & 0.906 & 0.007 & 0.894 \\
\hline & 2 & 0.036 & 0.491 & -0.001 & 0.985 & -0.001 & 0.978 \\
\hline & 3 & 0.032 & 0.547 & -0.003 & 0.951 & 0.003 & 0.953 \\
\hline \multirow[t]{3}{*}{ IL-1RA } & 1 & 0.140 & 0.006 & 0.131 & 0.011 & -0.129 & 0.012 \\
\hline & 2 & 0.172 & 0.004 & 0.157 & 0.011 & -0.111 & 0.068 \\
\hline & 3 & 0.163 & 0.008 & 0.152 & 0.014 & -0.100 & 0.104 \\
\hline \multirow[t]{3}{*}{ Adiponectin } & 1 & 0.020 & 0.716 & 0.053 & 0.340 & -0.038 & 0.490 \\
\hline & 2 & 0.011 & 0.861 & 0.056 & 0.362 & -0.062 & 0.307 \\
\hline & 3 & 0.010 & 0.873 & 0.063 & 0.313 & -0.060 & 0.326 \\
\hline
\end{tabular}

Data are given as standardised regression coefficients $\beta$ from linear regression analyses and corresponding $P$ values. Bold print indicates nominally significant associations with $P<0.05$. Circulating levels of biomarkers of subclinical inflammation (measured in the units as listed in Table 1) entered the models as In-transformed variables.

Model 1: adjusted for age, sex and study.

Model 2: model $1+\mathrm{BMl}, \mathrm{HbA} 1 \mathrm{c}$, time since diagnosis of diabetes, total cholesterol, triglycerides, use of lipid-lowering drugs, hypertension, use of NSAIDs, use of antithrombotic medication, use of antidepressant medication. Model 3: model 2 + number of diabetes-related comorbidities.

but differed in their strength by the measurements that were used to assess depressive symptoms.

\section{hsCRP, IL-6 and depressive symptoms}

The acute-phase protein CRP and the proinflammatory cytokine IL- 6 are the most frequently investigated biomarkers of inflammation in cross-sectional and prospective studies on depression. Several meta-analyses demonstrated positive associations between both proteins, depressive symptoms and major depression in community- or population-based samples ${ }^{20,21,37}$.

This study found associations between higher hsCRP and depressive symptoms assessed by one of the three scores in patients with T1D (WHO-5) and T2D (PHQ-9). The relationship between hsCRP and depressive symptoms has been analysed before in children and adolescents
Table 4 Associations between biomarkers of subclinical inflammation and depression scores in individuals with T2D

\begin{tabular}{|c|c|c|c|c|c|c|c|}
\hline \multirow[t]{2}{*}{ Biomarker } & \multirow[t]{2}{*}{ Model } & \multicolumn{2}{|l|}{ CES-D } & \multicolumn{2}{|l|}{ PHQ-9 } & \multicolumn{2}{|l|}{ WHO-5 } \\
\hline & & $\beta$ & $P$ & $\beta$ & $P$ & $\beta$ & $P$ \\
\hline \multirow[t]{3}{*}{ hsCRP } & 1 & 0.122 & 0.090 & 0.113 & 0.121 & -0.116 & 0.121 \\
\hline & 2 & 0.115 & 0.165 & 0.163 & 0.051 & -0.071 & 0.401 \\
\hline & 3 & 0.116 & 0.163 & 0.165 & 0.048 & -0.071 & 0.402 \\
\hline \multirow[t]{3}{*}{ IL-6 } & 1 & 0.035 & 0.614 & -0.002 & 0.973 & -0.009 & 0.907 \\
\hline & 2 & 0.032 & 0.670 & 0.036 & 0.634 & 0.010 & 0.900 \\
\hline & 3 & 0.033 & 0.663 & 0.038 & 0.610 & 0.011 & 0.884 \\
\hline \multirow[t]{3}{*}{ IL-18 } & 1 & 0.095 & 0.164 & 0.107 & 0.122 & -0.161 & 0.022 \\
\hline & 2 & 0.115 & 0.107 & 0.166 & 0.020 & -0.197 & 0.007 \\
\hline & 3 & 0.113 & 0.113 & 0.163 & 0.023 & -0.196 & 0.007 \\
\hline \multirow[t]{3}{*}{ CCL2 } & 1 & -0.043 & 0.536 & -0.071 & 0.315 & 0.015 & 0.841 \\
\hline & 2 & -0.031 & 0.667 & -0.057 & 0.425 & 0.012 & 0.872 \\
\hline & 3 & -0.031 & 0.670 & -0.055 & 0.445 & 0.016 & 0.832 \\
\hline \multirow[t]{3}{*}{ IL-1RA } & 1 & 0.162 & 0.021 & 0.093 & 0.191 & -0.203 & 0.005 \\
\hline & 2 & 0.168 & 0.041 & 0.152 & 0.068 & -0.223 & 0.006 \\
\hline & 3 & 0.168 & 0.043 & 0.152 & 0.069 & -0.230 & 0.006 \\
\hline \multirow[t]{3}{*}{ Adiponectin } & 1 & -0.098 & 0.187 & -0.158 & 0.034 & 0.061 & 0.429 \\
\hline & 2 & -0.111 & 0.153 & -0.209 & 0.007 & 0.096 & 0.230 \\
\hline & 3 & -0.109 & 0.164 & -0.204 & 0.009 & 0.098 & 0.222 \\
\hline
\end{tabular}

Data are given as standardised regression coefficients $\beta$ from linear regression analyses and corresponding $P$ values. Bold print indicates nominally significant associations with $P<0.05$. Circulating levels of biomarkers of subclinical inflammation (measured in the units as listed in Table 1) entered the models as In-transformed variables.

Model 1: adjusted for age, sex and study.

Model 2: model $1+\mathrm{BMI}$, $\mathrm{HbA1C}$, time since diagnosis of diabetes, total cholesterol, triglycerides, use of lipid-lowering drugs, hypertension, use of NSAIDs, use of antithrombotic medication, use of antidepressant medication. Model 3: model 2 + number of diabetes-related comorbidities.

with T1D in the USA ${ }^{23}$. and in adult patients with recentonset T1D in Germany ${ }^{24}$, but results were conflicting. A recent report linking higher levels of hsCRP with a poorer response to depression treatment in patients with T1D argued in favour of clinical relevance of hsCRP in patients with depression and $\mathrm{T}_{1} \mathrm{D}^{46}$. For T2D our results confirm findings from other patient samples ${ }^{23-25,27}$. Collectively, it appears that associations between hsCRP and depressive symptoms are similar in individuals with and without diabetes and may be independent of diabetes type.

In contrast, we observed no associations between IL-6 levels and depressive symptoms in patients with T1D or T2D. This finding is mainly in line with previous studies in diabetes patients ${ }^{23,24,27}$. Thus, our study corroborates the notion that IL- 6 may not be associated with depressive symptoms in patients with diabetes and that diabetes may 
have an effect-modifying impact on the association seen in the general population ${ }^{20,37}$. Given the strong correlations between hsCRP and IL-6 levels this result appears surprising, and so far it is unclear which mechanisms may be responsible for this observation.

\section{IL-18, IL-1RA and depressive symptoms}

IL-18 and IL-1RA are members of the IL-1 cytokine family. IL-18 has proinflammatory activities, whereas IL$1 \mathrm{RA}$ is an counterregulator of the potent proinflammatory cytokine IL- $1 \beta^{40}$. IL-1 $\beta$ and IL-18 are released upon activation of the Nod-like receptor pyrin containing 3 (NLRP3) inflammasome. The activation of the NLRP3 inflammasome affects immune cells, insulin-responsive cells including pancreatic beta cells and cells within the central nervous system. Thus, it has not only been implicated in the development of T1D and T2D ${ }^{40,41}$, but has also been found in chronic stress models and patients with major depressive disorder ${ }^{19,42}$. Therefore, associations between IL-1 cytokine family members, diabetes and depressive symptoms are biologically plausible.

This study indeed found an association between higher IL-18 levels and depressive symptoms in T2D, but not in T1D. The positive association for IL-18 was observed for the PHQ-9 and WHO-5 scores, which have not been used before in relation to this cytokine in patients with T2D. The association failed to reach significance for CES-D as measure of depression, which is in line with the null finding of our previous study in patients with recent-onset T2D, which was also based on the CES-D score ${ }^{24}$. Our null findings for patients with T1D corroborate two previous reports ${ }^{23,24}$. Therefore, this study suggests a difference between $\mathrm{T} 1 \mathrm{D}$ and $\mathrm{T} 2 \mathrm{D}$ with respect to the association of IL-18 with depressive symptoms, but it was not adequately powered to investigate such a difference with a formal interaction test. However, the strong correlations between IL-18 and hsCRP, IL- 6 and IL-1RA in patients with T2D in contrast to their absence in T1D point towards differences in the regulation and/or the pathophysiological relevance of this cytokine between both diabetes types. The finding of a positive association of IL-18 with depressive symptoms in T2D is interesting, because this cytokine is also involved in the development of other diabetes-related comorbidities ${ }^{43,44}$.

A comparison with IL-18 data from the general population is difficult because a recent meta-analysis was inconclusive due to the overall low number and sample sizes of available studies and the high heterogeneity between studies ${ }^{38}$. However, there is increasing experimental evidence linking stress, IL-18 and depression and in a complex manner. Induction of sadness increased circulating IL-18 levels in individuals without and with depression, possibly mediated by $\mu$-opioid system activation, while neutral mood induction reduced IL-18 ${ }^{47-49}$.
Based on these data, higher IL-18 levels could be interpreted as marker for dysregulated emotion. Studies in mice demonstrated that knockout of IL-18 showed reduced depressive-like behaviour ${ }^{50}$ and that the induction of IL-18 in the basolateral amygdala by chronic stress resulted in depressive-like behaviour, which could be suppressed by local inhibition of the IL-18 system in the amygdala $^{51}$. Thus, IL-18 may play an important role in linking environmental stimuli and the development of depressive symptoms.

Our study also demonstrated that higher IL-1RA levels were associated with depressive symptoms in both T1D and T2D. The data for T1D are novel, because we are not aware of other studies which analysed IL-1RA in this context. Our results for T2D are in line with a study in patients from the $\mathrm{UK}^{27}$, with a meta-analysis of community-based or population-based studies ${ }^{20,52}$ and with a prospective study showing that higher IL-1RA levels were related to higher risk of developing depressed mood in older persons ${ }^{53}$. Thus, our study extends the evidence that systemic IL-1RA levels are upregulated in patients with depressive symptoms to patients with T1D. The difficulty to reliably measure circulating levels of IL$1 \alpha$ and IL- $1 \beta$ means that we could not investigate the ratio between these cytokines and IL-1RA as antagonist. IL-1 $\alpha$ acts mainly as intracellular cytokine, and serum or plasma levels of IL-1 $\beta$ are below the levels of detection for most individuals in the absence of major trauma or infection, when high-sensitivity ELISAs as gold-standard method for protein quantification are used ${ }^{40}$. Nevertheless, these data indicate that IL-1-related processes and compensatory anti-inflammatory mechanisms may be relevant in patients with depression irrespectively of their diabetes status as previously also shown for macro- and microvascular comorbidities of diabetes ${ }^{40,42,45}$.

\section{CCL2, adiponectin and depressive symptoms}

Data on associations between the chemokine CCL2 and the adipokine adiponectin with depression are relatively scarce. We observed no associations between CCL2 and depressive symptoms in T1D and T2D, but an inverse association between adiponectin and depressive symptoms in patients with T2D.

The data for CCL2 are novel because we are not aware of comparable analyses in patients with T1D. CCL2 levels have been found associated with depressive symptoms in one study in patients with $\mathrm{T} 2 \mathrm{D}^{27}$, which we could not replicate. A recent meta-analysis reported higher CCL2 levels in patients with major depression compared to healthy controls. However, it also demonstrated high heterogeneity between studies and provided evidence that this result might be attributable to substantial publication bias $^{22}$. CCL2 represents a relevant biomarker in the context of diabetes because higher systemic levels are 
associated with higher risk of other comorbidities and mortality $^{54}$, but its potential relevance for depression remains to be elucidated.

An association between lower adiponectin and more pronounced depressive symptoms has not been reported in other studies in $\mathrm{T} 2 \mathrm{D}^{23,24,27}$, whereas our null findings in T1D corroborate previous reports ${ }^{23,24}$. Data from a recent meta-analysis are in line with our result for T2D, because it suggests that adiponectin levels may be lower in patients with major depression compared to controls without depression. However, it also indicated that this difference may be partly explained by confounding factors ${ }^{39}$.

\section{Clinical relevance of our findings and implications for future studies}

The findings for IL- 6 emphasise that results may differ between individuals without and with diabetes, so that diabetes status should be investigated as potential effect modifier in future studies. The data from this study also suggest that associations between biomarkers of inflammation and depressive symptoms may be similar for some proteins (hsCRP, IL-1RA), but different for others (IL-18, adiponectin) when comparing patients with T1D and T2D. The number of prior studies that have investigated patients with both diabetes types in the same analysis is small $^{23,24}$, and all three studies (i.e., the aforementioned studies and this one) were based on patients who varied in age and diabetes duration. Nevertheless, the currently available evidence points towards the possibility that such differences between T1D and T2D exist and argues in favour of analyses stratified by diabetes type rather than joint analyses of diabetes patients in this context.

Finally, the use of three questionnaires to assess depressive symptoms was helpful to cross-validate results, but it is important to keep in mind that different instruments measure different patterns of depressive symptoms. It seems plausible that such differences are also reflected by variations in associations between biomarkers of inflammation and these scores. We observed two significant associations of biomarkers with the CES-D score, four with the PHQ-9 and three with the WHO-5 score. Although no biomarker was associated with all three scores it is noteworthy that in cases with two significant associations the third one had only slightly lower effect sizes and $P$ values between 0.069 and 0.113 , indicating that the lack of statistical significance was mainly due to sample size. Taken together, our findings highlight the need for precise descriptions of depression outcomes in biomarker studies and the difficulties in comparing or meta-analysing studies using different measurement scores and/or definitions of depression.

Thus, our study may help to inform future studies seeking to gain further insights into the relationship between inflammation, diabetes and depression.
Important questions for further research concern the direction of effects, the impact of immune activation on the response to depression therapy and the underlying molecular mechanisms which could be targeted. There is evidence that depression may cause inflammation and vice versa because anti-depressant therapy has been reported to decrease systemic levels of inflammationrelated biomarkers ${ }^{55,56}$, while anti-inflammatory agents may alleviate depressive symptoms ${ }^{57,58}$. In addition to this potentially bidirectional relationship, both inflammation and depression likely share biological pathways ${ }^{11,13,14,59}$ such as the aforementioned activation of the NLRP3 inflammasome ${ }^{19,42}$, which adds to the complexity of the cross-talk between the immune system and the central nervous system, but may also present novel therapeutic opportunities $^{19,60,61}$.

\section{Strengths and limitations}

Strengths of our study are the large sample for association analyses, the inclusion of both patients with T1D and T2D, the use of three instruments to assess depressive symptoms and the measurement of six biomarkers reflecting different facets of immune activation. Additionally, the study participants were also well phenotyped with respect to potentially confounding factors such as comorbidities. Finally, we used few exclusion criteria for the study population to limit selection bias.

Limitations include the cross-sectional design and the sample size that was too limited for formal interaction tests to compare results between diabetes types or depression scores. Our focus on diabetes patients with subclinical depression and mild-to-moderate depression entailed the lack of inclusion of diabetes-free controls and depressionfree controls or patients with severe major depression. The fact that our study relied on patients with diabetes and subthreshold or mild forms of depression also meant that we had a reduced variance of depression severity due to exclusion of people without depressive symptoms and those with severe depression. Our study did not contain other inflammation-related biomarkers which might also have been of interest in this context (e.g., IL-1 $\beta$, IL-17, TNF $\alpha$ etc.), partly because of the aforementioned analytical reasons and partly because data implicating systemic levels of these biomarkers in the pathophysiology of diabetic complications are more limited than for the other selected biomarkers. However, a more comprehensive characterisation of subclinical inflammation by measurement of further biomarkers will undoubtedly contribute to a better understanding of its role for the development of depression in patients with T1D and T2D. We did not correct for multiple testing due to the strong correlations both among biomarkers and among depression scores, so that results should be considered exploratory. Due to different samples sizes of people with T1D and T2D, the statistical power to 
detect significant associations differed between both subsamples. Lastly, data may not be generalisable to diabetes patients with non-European descent.

\section{Conclusion}

This study showed differential associations between biomarkers of subclinical inflammation and depressive symptoms in patients with type 1 and type 2 diabetes rather than a general immune activation in diabetic individuals with depressive symptoms. These immune profiles show overlaps with risk factors for other comorbidies of diabetes. In conclusion, the results provide preliminary evidence for disease- and/or diabetes typespecific associations which may depend on the instrument used to assess depression.

\section{Acknowledgements}

This study was supported by the German Federal Ministry of Education and Research (BMBF) (FKZ 01GI0809 and FKZ 01Gl1107). The German Diabetes Center is funded by the German Federal Ministry of Health (BMG) and the Ministry of Innovation, Science, Research and Technology (MIWF) of the State North Rhine-Westphalia. This study was also supported in part by a grant from the German Federal Ministry of Education and Research (BMBF) to the German Center for Diabetes Research (DZD e.V.). The funders had no role in study design, data collection, data analysis, data interpretation, writing of the report and decision to publish the manuscript. We appreciate the voluntary contribution of all study participants. We also thank Thomas Weber and Silke Balbach (Bad Mergentheim, Germany) for excellent technical assistance and taking care of the patients. We further thank Ulrike Partke (DDZ, Düsseldorf, Germany), for excellent technical assistance.

\section{Author details}

${ }^{1}$ Institute for Clinical Diabetology, German Diabetes Center, Leibniz Center for Diabetes Research at Heinrich Heine University Düsseldorf, Düsseldorf, Germany. ${ }^{2}$ German Center for Diabetes Research (DZD), München-Neuherberg, Germany. ${ }^{3}$ Research Institute of the Diabetes Academy Mergentheim (FIDAM), Bad Mergentheim, Germany. ${ }^{4}$ Department for Psychology, Otto Friedrich University of Bamberg, Bamberg, Germany. ${ }^{5}$ Division of Endocrinology and Diabetology, Medical Faculty, Heinrich Heine University Düsseldorf, Düsseldorf, Germany

\section{Competing interests}

The authors declare that they have no competing financial interests.

Publisher's note: Springer Nature remains neutral with regard to jurisdictional claims in published maps and institutional affiliations.

Received: 2 March 2017 Revised: 17 August 2017 Accepted: 20 August 2017 Published online: 08 December 2017

\section{References}

1. Nouwen, A. et al. Type 2 diabetes mellitus as a risk factor for the onset of depression: a systematic review and meta-analysis. Diabetologia $\mathbf{5 3}$ 2480-2486 (2010).

2. Roy, T. \& Lloyd, C. E. Epidemiology of depression and diabetes: a systematic review. J. Affect. Disord. 142, S8-S21 (2012).

3. Buchberger, B. et al. Symptoms of depression and anxiety in youth with type 1 diabetes: a systematic review and meta-analysis. Psychoneuroendocrinology 70, 70-84 (2010).

4. Meurs, M. et al. Association of depressive and anxiety disorders with diagnosed versus undiagnosed diabetes: an epidemiological study of 90,686 participants. Psychosom. Med. 78, 233-241 (2016).
5. Pouwer, F. Depression: a common and burdensome complication of diabetes that warrants the continued attention of clinicians, researchers and healthcare policy makers. Diabetologia 60, 30-34 (2017).

6. Black, S. A. Markides, K. S. \& Ray, L. A. Depression predicts increased incidence of adverse health outcomes in older Mexican Americans with type 2 diabetes. Diabetes Care 26, 2822-2828 (2003).

7. Lin, E. H. et al. Depression and advanced complications of diabetes: a prospective cohort study. Diabetes Care 33, 264-269 (2010).

8. Sullivan, M. D. et al. Depression predicts all-cause mortality: epidemiological evaluation from the ACCORD RQL substudy. Diabetes Care 35, 1708-1715 (2010).

9. Pouwer, F., Nefs, G. \& Nouwen, A. Adverse effects of depression on glycemic control and health outcomes in people with diabetes: a review. Endocrinol. Metab. Clin. North Am. 42, 529-544 (2013).

10. van Dooren, F. E. et al. Depression and risk of mortality in people with diabetes mellitus: a systematic review and meta-analysis. PLoS ONE 8, e57058 (2013).

11. Korczak, D. J., Pereira, S., Koulajian, K., Matejcek, A. \& Giacca, A. Type 1 diabetes mellitus and major depressive disorder: evidence for a biological link. Diabetologia 54, 2483-2493 (2011)

12. Stuart, M. J. \& Baune, B. T. Depression and type 2 diabetes: inflammatory mechanisms of a psychoneuroendocrine co-morbidity. Neurosci. Biobehav. Rev. 36, 658-676 (2012).

13. Holt, R. I. et al. NIDDK international conference report on diabetes and depression: current understanding and future directions. Diabetes Care 37, 2067-2077 (2014).

14. Moulton, C. D., Pickup, J. C. \& Ismail, K. The link between depression and diabetes: the search for shared mechanisms. Lancet Diabetes Endocrinol. 3 461-471 (2015).

15. Leslie, R. D., Palmer, J., Schloot, N. C. \& Lernmark, A. Diabetes at the crossroads: relevance of disease classification to pathophysiology and treatment. Diabetologia 59, 13-20 (2016).

16. Skyler, J. S. et al. Differentiation of diabetes by pathophysiology, natural history, and prognosis. Diabetes 66, 241-255 (2017).

17. Dantzer, R., O'Connor, J. C., Freund, G. G., Johnson, R. W. \& Kelley, K. W. From inflammation to sickness and depression: when the immune system subjugates the brain. Nat. Rev. Neurosci. 9, 46-56 (2008).

18. Slavich, G. M. \& Irwin, M. R. From stress to inflammation and major depressive disorder: a social signal transduction theory of depression. Psychol. Bull. 140, 774-815 (2014)

19. Miller, A. H. \& Raison, C. L. The role of inflammation in depression: from evolutionary imperative to modern treatment target. Nat. Rev. Immunol. 16, 22-34 (2016).

20. Howren, M. B., Lamkin, D. M. \& Suls, J. Associations of depression with C-reactive protein, IL-1, and IL-6: a meta-analysis. Psychosom. Med. 71, 171-186 (2009).

21. Valkanova, V., Ebmeier, K. P. \& Allan, C. L. CRP, IL-6 and depression: a systematic review and meta-analysis of longitudinal studies. J. Affect. Disord. 150, 736-744 (2013).

22. Eyre, H. A. et al. A meta-analysis of chemokines in major depression. Prog. Neuropsychopharmacol. Biol. Psychiatry 68, 1-8 (2016).

23. Hood, K. K. et al. Metabolic and inflammatory links to depression in youth with diabetes. Diabetes Care 35, 2443-2446 (2012).

24. Herder, C. et al. Associations between inflammation-related biomarkers and depressive symptoms in individuals with recently diagnosed type 1 and type 2 diabetes. Brain Behav. Immun. 61, 137-145 (2017).

25. Hayashino, Y., Mashitani, T., Tsujii, S. \& Ishii, H., Diabetes Distress and Care Registry at Tenri Study Group. Elevated levels of hs-CRP are associated with high prevalence of depression in Japanese patients with type 2 diabetes: the Diabetes Distress and Care Registry at Tenri (DDCRT 6). Diabetes Care 37, 2459-2465 (2014).

26. Doyle, T. A. et al. Diabetes, depressive symptoms, and inflammation in older adults: results from the health, aging, and body composition study. J. Psychosom. Res. 75, 419-424 (2013).

27. Laake, J. P. et al. The association between depressive symptoms and systemic inflammation in people with type 2 diabetes: findings from the South London Diabetes Study. Diabetes Care 37, 2186-2192 (2014).

28. Hermanns, N. et al. The effect of a Diabetes-specific cognitive behavioral treatment program (DIAMOS) for patients with diabetes and subclinical depression: results of a randomized controlled trial. Diabetes Care 38, 551-560 (2015).

29. Andrews, G. \& Peters, L. The psychometric properties of the composite international diagnostic interview. Soc. Psychiatry Psychiatr. Epidemiol. 33, 80-88 (1998) 
30. Hautzinger, M. Die CES-D Skala: ein Depressionsmeßinstrument für Untersuchungen in der Allgemeinbevölkerung. Diagnostica 34, 167-173 (1988).

31. Hautzinger M., Bailer M., Hofmeister D., Keller F. ADS: Allgemeine Depressionsskala (2., überarbeitete und neu normierte Auflage) (ADS: General depression inventory 2nd revised, newly standardised edition) (in German) (Hogrefe, 2012).

32. Radloff, L. S. The CES-D scale. A self-report depression scale for research in the general population. Appl. Psychol. Measure 1, 385-401 (1977).

33. Vilagut, G., Forero, C. G., Barbaglia, G. \& Alonso, J. Screening for depression in the general population with the Center for Epidemiologic Studies Depression (CES-D); a systematic review and meta-analysis. PLOS ONE 11, e0155431 (2016).

34. Kroenke, K. Spitzer, R. L. \& Williams, J. B. The PHQ-9: validity of a brief depression severity measure. J. Gen. Intern. Med. 16, 606-613 (2001).

35. Topp, C. W., Østergaard, S. D., Søndergaard, S. \& Bech, P. The WHO-5 WellBeing Index: a systematic review of the literature. Psychother. Psychosom. 84, 167-176 (2015).

36. Herder, C. et al. Association of subclinical inflammation with polyneuropathy in the older population: KORA F4 study. Diabetes Care 36, 3663-3670 (2013).

37. Haapakoski, R., Mathieu, J., Ebmeier, K. P., Alenius, H. \& Kivimäki, M. Cumulative meta-analysis of interleukins 6 and $1 \beta$, tumour necrosis factor $a$ and $C$-reactive protein in patients with major depressive disorder. Brain Behav. Immun. 49 206-215 (2015).

38. Köhler, C. A. et al. Peripheral cytokine and chemokine alterations in depression: a meta-analysis of 82 studies. Acta Psychiatr. Scand. 135, 373-387 (2017).

39. Carvalho, A. F. et al. Adipokines as emerging depression biomarkers: a systematic review and meta-analysis. J. Psychiatr. Res. 59, 28-37 (2014).

40. Herder, C., Dalmas, E., Böni-Schnetzler, M. \& Donath, M. Y. The IL-1 pathway in type 2 diabetes and cardiovascular complications. Trends Endocrinol. Metab. 26, 551-563 (2015).

41. Hu, C. et al. NLRP3 deficiency protects from type 1 diabetes through the regulation of chemotaxis into the pancreatic islets. Proc. Natl Acad. Sci. USA 112, 11318-11323 (2015).

42. Kaufmann, F. N. et al. NLRP3 inflammasome-driven pathways in depression: clinical and preclinical findings. Brain. Behav. Immun. 64, 367-383 (2017).

43. Kaptoge, S. et al. Inflammatory cytokines and risk of coronary heart disease: new prospective study and updated meta-analysis. Eur. Heart J. 35, 578-589 (2014).

44. Herder, C. et al. Inflammatory markers are associated with cardiac autonomic dysfunction in recent-onset type 2 diabetes. Heart 103, 63-70 (2017).

45. Herder, C. et al. Circulating levels of interleukin 1-receptor antagonist and risk of cardiovascular disease: meta-analysis of six population-based cohorts. Arterioscler. Thromb. Vasc. Biol. 37, 1222-1227 (2017).

46. Zahn, D. et al. hs-CRP predicts improvement in depression in patients with type 1 diabetes and major depression undergoing depression treatment: results from the Diabetes and Depression (DAD) Study. Diabetes Care 39, e171-e173 (2016).
47. Prossin, A. R. et al. Association of plasma interleukin-18 levels with emotion regulation and $\mu$-opioid neurotransmitter function in major depression and healthy volunteers. Biol. Psychiatry 69, 808-812 (2011).

48. Prossin, A. R. et al. Experimental sadness induces relevant interactions between central endogenous opioid activation and plasma IL-18 concentrations in depressed volunteers. Mol. Psychiatry 21, 151 (2016).

49. Prossin, A. R. et al. Acute experimental changes in mood state regulate immune function in relation to central opioid neurotransmission: a model of human CNS-peripheral inflammatory interaction. Mol. Psychiatry 21, 243-251 (2016).

50. Yamamoto, Y. et al. Interleukin-18 deficiency reduces neuropeptide gene expressions in the mouse amygdala related with behavioral change. J. Neuroimmunol. 229, 129-139 (2010).

51. Kim, T. K. et al. Local interleukin-18 system in the basolateral amygdala regulates susceptibility to chronic stress. Mol. Neurobiol. 54, 5347-5358 (2017).

52. Lehto, S. M. et al. Serum anti-inflammatory markers in general population subjects with elevated depressive symptoms. Neurosci. Lett. 484, 201-205 (2010).

53. Milaneschi, Y. et al. Interleukin-1 receptor antagonist and incident depressive symptoms over 6 years in older persons: the InCHIANTI study. Biol. Psychiatry 65, 973-978 (2009).

54. Piemonti, L. et al. Association between plasma monocyte chemoattractant protein-1 concentration and cardiovascular disease mortality in middle-aged diabetic and nondiabetic individuals. Diabetes Care 32, 2105-2110 (2009).

55. Hiles, S. A., Baker, A. L., de Malmanche, T. \& Attia, J. Interleukin-6, C-reactive protein and interleukin-10 after antidepressant treatment in people with depression: a meta-analysis. Psychol. Med. 42, 2015-2026 (2012).

56. Strawbridge, R. et al. Inflammation and clinical response to treatment in depression: a meta-analysis. Eur. Neuropsychopharmacol. 25, 1532-1543 (2015).

57. Köhler, O. et al. Effect of anti-inflammatory treatment on depression, depressive symptoms, and adverse effects: a systematic review and metaanalysis of randomized clinical trials. JAMA Psychiatry 71, 1381-1391 (2014).

58. Kappelmann N., Lewis G., Dantzer R., Jones P. B., \& Khandaker G. M. Antidepressant activity of anti-cytokine treatment: a systematic review and metaanalysis of clinical trials of chronic inflammatory conditions. Mol. Psychiatry https://doi.org/10.1038/mp.2016.167 (2016)

59. Tabák, A. G., Akbaraly, T. N., Batty, G. D. \& Kivimäki, M. Depression and type 2 diabetes: a causal association? Lancet Diabetes Endocrinol. 2, 236-245 (2014).

60. Baumeister, D., Ciufolini, S. \& Mondelli, V. Effects of psychotropic drugs on inflammation: consequence or mediator of therapeutic effects in psychiatric treatment? Psychopharmacology 233, 1575-1589 (2016).

61. Köhler, O., Krogh, J., Mors, O. \& Benros, M. E. Inflammation in depression and the potential for anti-inflammatory treatment. Curr. Neuropharmacol. 14, 732-742 (2016) 